\title{
Rare incidence of primary adrenocortical carcinosarcoma: A case report and literature review
}

\author{
YONG-BAO WEI*, YUN-LIANG GAO*, HONG-TAO WU, SHI-FENG OU-YANG, \\ TAO XU, DONG-FANG MAO and JIN-RUI YANG
}

Department of Urology, The Second Xiangya Hospital, Central South University, Changsha, Hunan 410011, P.R. China

Received January 2, 2014; Accepted August 12, 2014

DOI: $10.3892 / 01.2014 .2635$

\begin{abstract}
Adrenocortical carcinoma (ACC) is a rare, but highly aggressive type of tumor with an incidence of one to two per million annually. Adrenocortical carcinosarcoma is an exceptional variant of ACC, which is characterized by the presence of histological regions of carcinoma and sarcoma. To date, to the best of our knowledge, there have only been 12 reported cases of adrenocortical carcinosarcoma. In the present study, a case of primary, non-functional adrenocortical carcinosarcoma is described, as well as a review of the literature to raise awareness of this particularly rare type of malignant neoplasm that is associated with a worse diagnosis and prognosis than adrenocortical carcinoma. In the present study, the patient underwent a laparoscopic left adrenalectomy and the tumor was dissected without complication from the left kidney. Microscopic observations showed the tumor comprised of epithelial and spindle cell components. The patient did not exhibit signs of tumor recurrence at the one-month follow-up. The potential diagnosis of adrenocortical carcinosarcoma must be considered when diagnosing adrenal malignancies in adults. In addition, comphrensive imunohistochemical staining may be required to identify possible sarcomatous patterns. To the best of our knowledge, the present case is the first to report an incidence of adrenocortical carcinosarcoma in China. Details of the patient are presented and the pathology of adrenocortical carcinosarcoma is discussed.
\end{abstract}

\section{Introduction}

Adrenocortical carcinoma (ACC) is a rare, but highly aggressive type of tumor with an incidence of one to two per

Correspondence to: Professor Jin-Rui Yang, Department of Urology, The Second Xiangya Hospital, Central South University, 139 Renmin Road, Changsha, Hunan 410011, P.R. China

E-mail: 2xiangya2010@gmail.com

*Contributed equally

Key words: adrenocortical carcinosarcoma, adrenocortical carcinoma, pathology million annually (1-3). Furthermore, a higher incidence of ACC has been reported in females. ACC is associated with a bimodal age distribution that has two peaks; one representing children in the first decade of life and the other representing adults in the fourth to fifth decades of life (4-5). A small number of cases of ACC have been reported to date, each with a poor prognosis and a five-year overall survival rate of $35 \%(4,6-8)$. The poor prognosis may be attributable in part to the advanced stage at which the majority of ACCs are detected (4).

Adrenocortical carcinosarcoma is an exceptional variant of ACC, which is characterized by the presence of histological regions of carcinoma and sarcoma, with the latter regularly demonstrating heterologous features, including rhabdomyoblastic (9-12), chondroid or osteogenic differentiation $(9,13)$. The term sarcomatoid carcinoma has also been synonymously used to refer to adrenocortical carcinosarcoma. In the present study, a case of primary, non-functional adrenocortical carcinosarcoma is described and a review of the literature is presented to raise awareness of this particularly rare type of malignant neoplasm with a poor diagnosis and prognosis. To the best of our knowledge, the present case is the first to report an incidence of adrenocortical carcinosarcoma in China. Written informed consent was obtained from the family of the patient.

\section{Case report}

Patient information. In November 2013, a 63-year-old female was admitted to the Second Xiangya Hospital (Changsha, China) with left flank pain, fatigue, decreased appetite and weight loss that had persisted for three months. These clinical symptoms led to the discovery of a 7-cm heterogeneous hypoechoic left adrenal mass on an abdominal ultrasound. On admission to the Department of Urology at the Second Xiangya Hospital, the results of the physical examination were normal and there was no palpable abdominal mass. The patient exhibited no clinical features associated with excessive steroid hormone or catecholamine levels and had no notable family medical history. Furthermore, laboratory studies of the adrenal hormone levels of cortisol, aldosterone and catecholamines were within the normal limits. 24-h urine volume was $1100 \mathrm{ml}$ (normal range, 1000-1500 ml), 24-h urinary vanillylmandelic acid level was $33.1 \mu \mathrm{mol} /$ day 

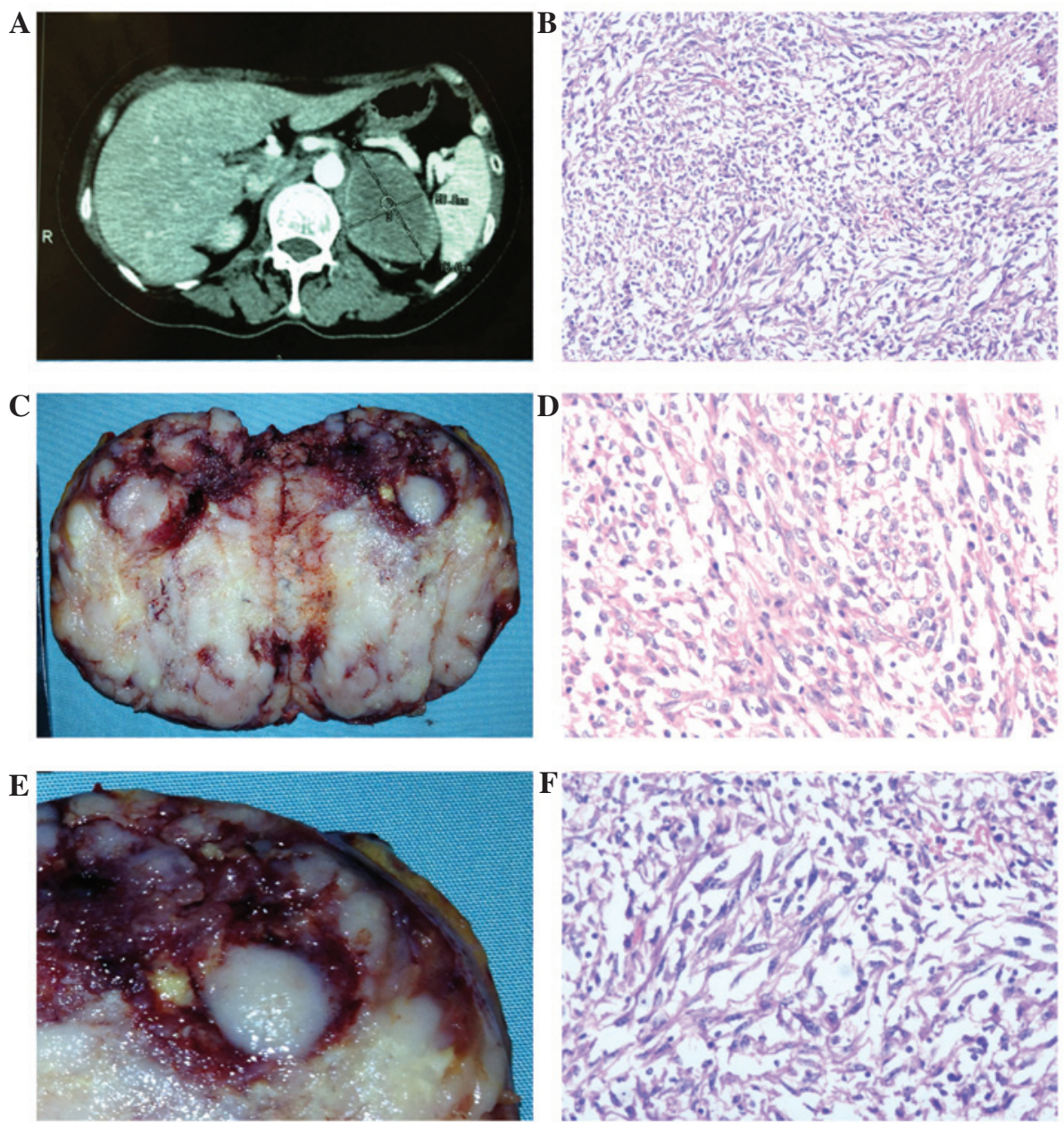

Figure 1. Gross images and hematoxylin and eosin (H\&E)-stained microscopic images of the primary adrenocortical carcinosarcoma. (A) A computed tomography scan demonstrates a 7.6x5.1-cm well-demarcated and peripherally enhanced left adrenal mass impinging on the pancreas and the spleen, without parenchymal invasion into the kidney. (B) Gross image of the cut surface of the adrenocortical carcinosarcoma shows a circumscribed mass, predominantly comprising paler, firm tissue, with partially yellow tissue. (C) A compressed rim of normal adrenal gland is apparent adjacent to the tumor capsule. (D) The tumor comprises of spindle cell and epithelial components (H\&E staining; magnification, x100). (E) Carcinomatous cells exhibit highly atypical nuclei and an abundant eosinophilic cytoplasm (H\&E staining; magnification, x200). (F) Spindle-shaped tumor cells reveal highly pleomorphic nuclei (H\&E staining; magnification, x200).
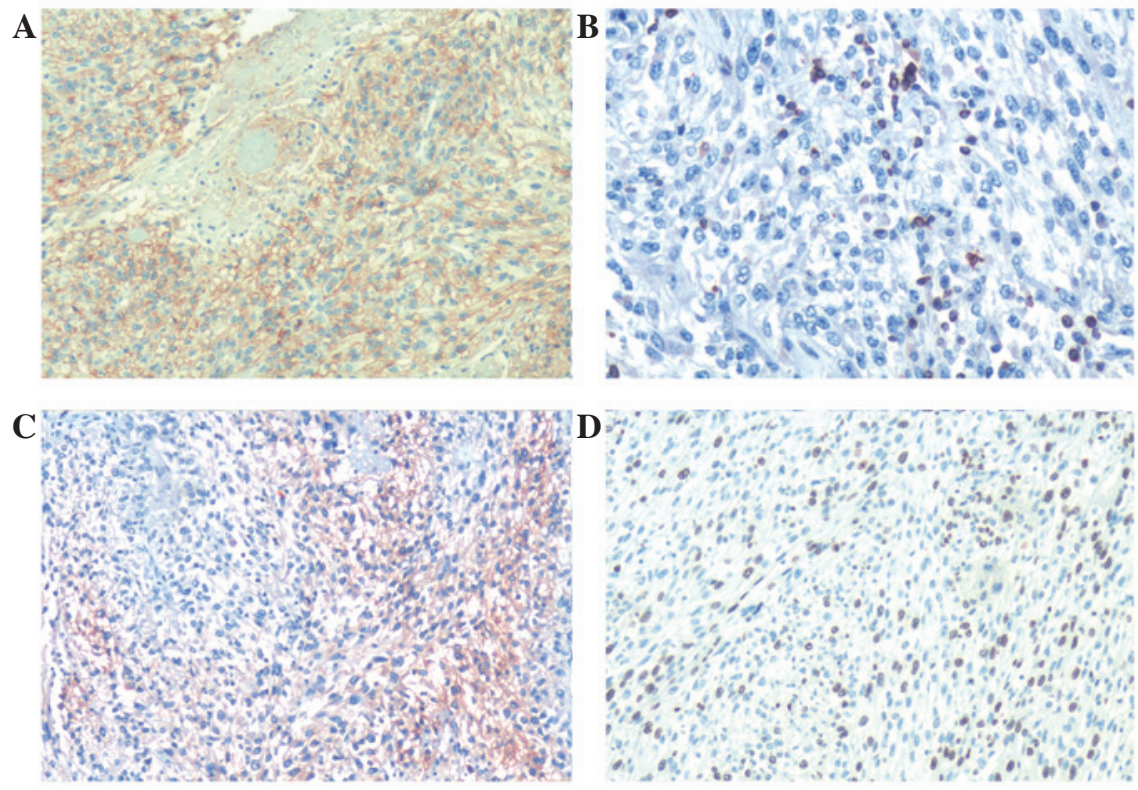

Figure 2. Immunohistochemical staining of the primary adrenocortical carcinosarcoma. (A) Carcinomatous and sarcomatous cells positive for cluster of differentiation 56 (magnification, x200). Cytoplasm of the sarcomatous cells positive for (B) Bcl-2 (magnification, x200) and (C) neuron specific enolase (magnification, x100). (D) Carcinomatous and sarcomatous cells positive for Ki-67 (x100). 


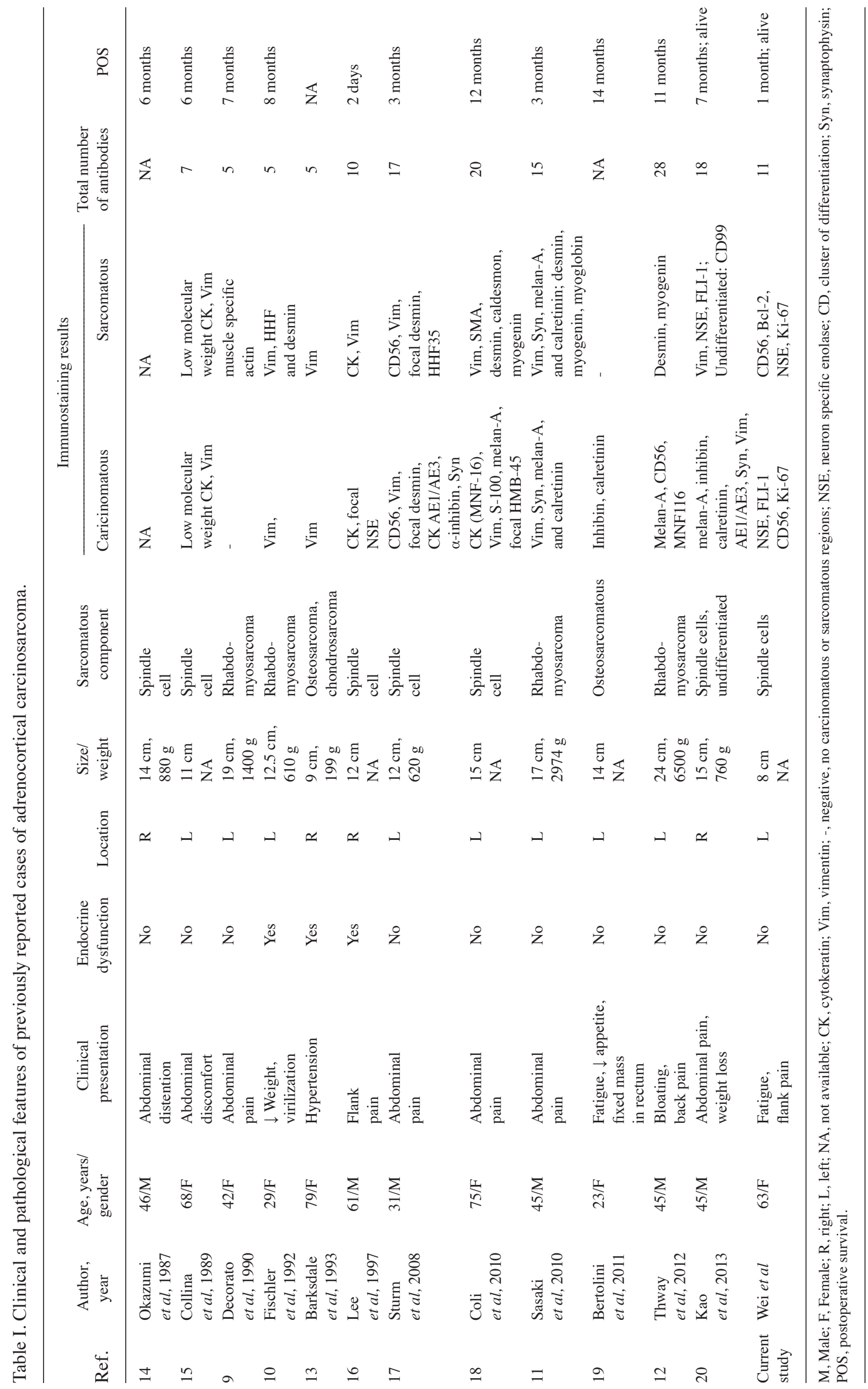


(normal range, 0-68.6 $\mu \mathrm{mol} / \mathrm{day}$ ), the clinostatic and orthostatic plasma renin activity levels were $270 \mathrm{ng} / \mathrm{l} / \mathrm{h}$ (normal range, $150-2330 \mathrm{ng} / \mathrm{l} / \mathrm{h}$ ) and $388 \mathrm{ng} / \mathrm{l} / \mathrm{h}$ (normal range, 100-6560 ng/l/h), respectively. The clinostatic and orthostatic plasma aldosterone levels were $56 \mathrm{ng} / \mathrm{l}$ (normal range, 30-160 ng/l) and $119 \mathrm{ng} / \mathrm{l}$ (normal range, 70-300 ng/l), respectively. A dynamic, contrast-enhanced abdominal computed tomography scan showed a $7.6 \times 5.1-\mathrm{cm}$ well-demarcated and peripherally enhanced left adrenal mass that was impinging on the pancreas and the spleen, without parenchymal invasion into the kidney (Fig. 1A). Distant lymph node, pulmonary or liver metastases were not observed. A laparoscopic left adrenalectomy was performed and the tumor was dissected without complication from the left kidney. The patient did not exhibit signs of tumor recurrence at the one-month follow-up.

Gross pathology. The gross specimen presented as a well-demarcated, encapsulated spherical mass, which measured $8 \times 6 \times 4 \mathrm{~cm}$. The cut surface was firm and varied in color. The majority of the lesion was pale, however, certain regions ( $\sim 30 \%$ of the tumor area) were bright yellow (Fig. 1B). Approximately $20 \%$ of the tumor area comprised of numerous foci of necrosis. There was a small quantity of the residual adrenal gland, which appeared to be laminated at the periphery of the tumor (Fig. 1C). The spleen, pancreas and kidney had not been invaded by the tumor.

Microscopic observations. The tumor comprised of epithelial and spindle cell components that were multifocally intermingled (Fig. 1D and E). In addition, focal necrosis and hemorrhaging was observed. The main epithelial component exhibited poorly differentiated carcinomatous cells, which were arranged in a diffuse and solid growth pattern. These cells showed highly atypical nuclei, and an abundant eosinophilic cytoplasm and usually possessed large, prominent nucleoli (Fig. 1E). Furthermore, spindle-shaped tumor cells were observed to be arranged in a haphazard fascicular pattern with highly pleomorphic nuclei (Fig. 1F).

Variable numbers of cells undergoing mitosis were identified in different areas. The highest mitotic activity was observed in the sarcomatous area, however, only occasional instances of mitosis were observed in the carcinomatous area. Furthermore, no definite capsular invasion was observed histologically.

Immunohistochemical findings. An extensive immunohistochemical panel was performed on formalin-fixed, paraffin-embedded tumor tissue to evaluate the sarcomatous and carcinomatous components. In addition, negative and positive controls were performed for each immunohistochemical stain, including cytokeratin (CK), synapsin (Syn), CD56, CD34, neuron-specific enolase (NSE), Ki-67, S100, smooth muscle actin, Bcl-2, human melanoma black 45 (HMB-45) and chromogranin $\mathrm{A}(\mathrm{CgA})$. Negative controls included primary antigens (rabbit anti-human antibodies), while positive control included the aforementioned primary antigens and the secondary (goat anti-rabbit) antibodies. Cluster of differentiation (CD)56 demonstrated diffuse positivity in the epithelial cells and spindle areas (Fig. 2A). Bcl-2 showed focal positivity in the sarcomatous areas (Fig. 2B) and CD34 was weakly positive. NSE was only weakly positive in sarcomatous components (Fig. 2C) and Ki-67 demonstrated $70 \%$ positivity in the two components (Fig. 2D).
Table II. Immunohistochemical results (not including cytokeratins) of the reported adrenocortical carcinosarcoma.

\begin{tabular}{lccc} 
A, Carcinomatous component & & \\
\hline Antibody & Check sum & Positive & Positive rate \\
\hline FLI-1 & 1 & 1 & 100 \\
Melan-A & 5 & 4 & 80 \\
Vimentin & 9 & 7 & 78 \\
CD56 & 4 & 3 & 75 \\
Inhibin & 6 & 3 & 50 \\
Ki-67 & 2 & 1 & 50 \\
Syn & 7 & 3 & 43 \\
NSE & 5 & 2 & 40 \\
HMB-45 & 5 & 1 & 20 \\
Desmin & 7 & 1 & 14 \\
S-100 & 8 & 1 & 13 \\
\hline
\end{tabular}

$\mathrm{B}$, Sarcomatous component

\begin{tabular}{lccc}
\hline Antibody & Check sum & Positive & Positive rate \\
\hline HHF & 3 & 3 & 100 \\
Bcl-2 & 1 & 1 & 100 \\
FLI-1 & 1 & 1 & 100 \\
Vimentin & 9 & 8 & 89 \\
Desmin & 7 & 5 & 71 \\
CD56 & 4 & 2 & 50 \\
Caldesmon & 2 & 1 & 50 \\
Ki-67 & 2 & 1 & 50 \\
Myoglobin & 2 & 1 & 50 \\
Myogenin & 5 & 2 & 40 \\
NSE & 5 & 2 & 40 \\
Calretinin & 5 & 1 & 20 \\
Melan-A & 5 & 1 & 20 \\
SMA & 6 & 1 & 17 \\
Syn & 7 & 1 & 14 \\
\hline
\end{tabular}

$\mathrm{CD}$, cluster of differentiation; Syn, synaptophysin; NSE, neuron specific enolase; HMB-45, human melanoma black 45; SMA, smooth muscle actin.

S-100 showed equal amounts of positivity/negativity, and CK, Syn, smooth muscle actin, HMB-45 and CgA showed no reactivity in all of the areas.

\section{Discussion}

ACCs containing a component of sarcoma or sarcoma-like (spindle cells) differentiation are a particularly rare and aggressive type of neoplasm, and, to the best of our knowledge, only 12 cases have previously been reported in the English literature to date (9-20). The clinical and pathological features of reported patients presenting with this type of tumor, including the present case, are summarized in Table I (9-20). The age of initial presentation ranged from 23 to 79 years, with a mean of 50.3 years and a median of 45 years, which appears to be similar to the results of a previous study (mean age, 40-50 years) by Sasaki et al (11). Eight of the 13 patients were aged $<50$ years. 
Compared with previous studies of ACC (4), adrenocortical carcinosarcoma also showed a female preponderance with a female/male ratio of 1.6:1. Flank/abdominal pain or discomfort was identified to be a common presentation (10/13 cases) and the majority of adrenocortical carcinosarcoma presented in the left adrenal gland (9/12 cases). One tumor was identified during an investigation of a rectal mass in a pregnant patient (19). In the majority of cases, these tumors did not exhibit any endocrine dysfunction, although three of the 13 cases were associated with corticosteroid hypersecretion $(10,13,16)$. Generally, the tumors were particularly large (mean size, $14.1 \mathrm{~cm}$; weight, 1,743 g) and exhibited dramatically aggressive behavior. All 13 patients succumbed to their cancer within the range of two days to 14 months following resection, despite aggressive administration of multimodality therapeutic strategies.

The present case was extensively sampled, however, the heterologous elements, such as rhabdomyosarcoma (9) and osteosarcoma (13), that were documented in certain previous papers were not observed. Additionally, when compared with previous studies, in this study there were no specific immunohistochemical staining results. The positive reactivity with NSE that was only observed in partial sarcomatous areas may provide evidence for neuroendocrine differentiation in ACCs, which is consistent with two previous studies; one demonstrated positive staining for NSE, Syn and neurofilament protein (21), and the other showed positivity for CK AE1/AE3, Syn and NSE (20).

Notably, the tumor mass observed in the present case, (size, $8 \times 6 \times 4 \mathrm{~cm}$ ) was the smallest out of all of the reported cases. The next smallest, measuring $9 \times 7.5 \times 6.5 \mathrm{~cm}$, was reported in 1993 (13). The tumor sizes described in the other 11 cases were all $>10 \mathrm{~cm}$, which indicates that although a tumor mass may not be large it may be an adrenocortical carcinosarcoma. Therefore, the diagnosis of adrenocortical carcinosarcoma may be complicated.

Furthermore, a compressed rim of normal adrenal gland was observed adjacent to the tumor capsule in the present study, which was also described in two previous cases $(12,20)$ and in a case of large diameter ACC (22). Therefore it may be hypothesized that the tumor arises from an accessory/ectopic adrenal gland, or alternatively, from a nodular area of the adrenal gland, which gradually becomes entirely replaced by the tumor (22).

A challenge during the diagnosis of adrenocortical carcinosarcoma arises from the difficulty in grossly identifying the tumor origin. Renal carcinosarcoma, metastatic melanoma and primary retroperitoneal sarcoma should be considered in the differential diagnosis, and attention should be given to ACCs demonstrating negative, or only focally weak, positivity for CKs. Consequently, it is necessary to adopt immunohistochemical staining for melan-A, inhibin and calretinin to verify the adrenocortical origin, particularly for ACC (23-26). Routine histological data from Table II revealed that the carcinomatous and sarcomatous components expressed vimentin. A notable feature is that desmin was found to be highly expressed in the sarcomatous component. Furthermore, the sarcomatous component of the tumor was positive for HHF, myogenin, and caldesmon, whereas the epithelial component was diffusely positive for inhibin, melan-A, S-100 protein, NSE and HMB-45. As a result, a large number of tumor samples and an extensive panel of immunohistochemical staining are considered to be necessary to identify the adrenal origin and the biphasic components of adrenocortical carcinosarcoma.

In conclusion, a potential diagnosis of adrenocortical carcinosarcoma should be considered when diagnosing an adrenal malignancy in adult patients. Extensive sampling of the tumor together with comprehensive immunohistochemical staining are required to identify a possible sarcomatous pattern and, as a result of the poor prognosis associated with adrenocortical carcinosarcoma, the most aggressive therapeutic regimens are required.

\section{Acknowledgements}

The present study was supported by the Fundamental Research Funds for the Central Universities of Central South University in 2013 (grant no. 2013zzts095).

\section{References}

1. Lacroix A: Approach to the patient with adrenocortical carcinoma. J Clin Endocrinol Metab 95: 4812-4822, 2010.

2. Zini L, Porpiglia F and Fassnacht M: Contemporary management of adrenocortical carcinoma. Eur Urol 60: 1055-1065, 2011.

3. Fassnacht M, Libé R, Kroiss M and Allolio B: Adrenocortical carcinoma: a clinician's update. Nat Rev Endocrinol 7: 323-335, 2011.

4. Ng L and Libertino JM: Adrenocortical carcinoma: diagnosis, evaluation and treatment. J Urol 169: 5-11, 2003.

5. Roman S: Adrenocortical carcinoma. Curr Opin Oncol 18: 36-42, 2006.

6. Crucitti F, Bellantone R, Ferrante A, et al: The Italian Registry for Adrenal Cortical Carcinoma: analysis of a multiinstitutional series of 129 patients. The ACC Italian Registry Study Group. Surgery 119: 161-170, 1996.

7. Icard $\mathrm{P}$, Goudet $\mathrm{P}$, Charpenay $\mathrm{C}$, et al: Adrenocortical carcinomas: surgical trends and results of a 253-patient series from the French Association of Endocrine Surgeons study group. World J Surg 25: 891-897, 2001.

8. Tauchmanovà L, Colao A, Marzano LA, et al: Andrenocortical carcinomas: twelve-year prospective experience. World J Surg 28: 896-903, 2004.

9. Decorato JW, Gruber H, Petti M and Levowitz BS: Adrenal carcinosarcoma. J Surg Oncol 45: 134-136, 1990.

10. Fischler DF, Nunez C, Levin HS, et al: Adrenal carcinosarcoma presenting in a woman with clinical signs of virilization. A case report with immunohistochemical and ultrastructural findings. Am J Surg Pathol 16: 626-631, 1992.

11. Sasaki K, Desimone M, Rao HR, et al: Adrenocortical carcinosarcoma: a case report and review of the literature. Diagn Pathol 5: 51, 2010.

12. Thway K, Olmos D, Shah C, et al: Oncocytic adrenal cortical carcinosarcoma with pleomorphic rhabdomyosarcomatous metastases. Am J Surg Pathol 36: 470-477, 2012.

13. Barksdale SK, Marincola FM and Jaffe G: Carcinosarcoma of the adrenal cortex presenting with mineralocorticoid excess. Am J Surg Pathol 17: 941-945, 1993.

14. Okazumi S, Asano T, Ryu M, et al: Surgical resection of adrenal carcinoma extending into the vena cava, right atrium and ventricle: case report and review of the literature. Nihon Geka Gakkai Zasshi 88: 231-238, 1987 (In Japanese).

15. Collina G, Maldarizzi F, Betts CM and Eusebi V: Primary sarcomatoid carcinoma of the adrenal gland. First case report. Virchows Arch A Pathol Anat Histopathol 415: 161-167, 1989.

16. Lee MS, Park IA, Chi JG, et al: Adrenal carcinosarcoma - a case report. J Korean Med Sci 12: 374-377, 1997.

17. Sturm N, Moulai N, Laverrière MH, et al: Primary adrenocortical sarcomatoid carcinoma: case report and review of literature. Virchows Arch 452: 215-219, 2008.

18. Coli A, Di Giorgio A, Castri F, et al: Sarcomatoid carcinoma of the adrenal gland: a case report and review of literature. Pathol Res Pract 206: 59-65, 2010.

19. Bertolini F, Rossi G, Fiocchi F, et al: Primary adrenal gland carcinosarcoma associated with metastatic rectal cancer: a hitherto unreported collision tumor. Tumori 97: 27e-30e, 2011. 
20. Kao CS, Grignon DJ, Ulbright TM and Idrees MT: A case report of adrenocortical carcinosarcoma with oncocytic and primitive neuroectodermal-like features. Hum pathol 44: 1947-1955, 2013.

21. Miettinen M: Neuroendocrine differentiation in adrenocortical carcinoma. New immunohistochemical findings supported by electron microscopy. Lab Invest 66: 169-174, 1992.

22. Hoang MP, Ayala AG and Albores-Saavedra J: Oncocytic adrenocortical carcinoma: a morphologic, immunohistochemical and ultrastructural study of four cases. Mod Pathol 15: 973-978, 2002.

23. Jorda M, De MB and Nadji M: Calretinin and inhibin are useful in separating adrenocortical neoplasms from pheochromocytomas. Appl Immunohistochem Mol Morphol 10: 67-70, 2002 .
24. Ghorab Z, Jorda M, Ganjei P and Nadji M: Melan A (A103) is expressed in adrenocortical neoplasms but not in renal cell and hepatocellular carcinomas. Appl Immunohistochem Mol Morphol 11: 330-333, 2003.

25. Zhang PJ, Genega EM, Tomaszewski JE, et al: The role of calretinin, inhibin, melan-A, BCL-2, and C-kit in differentiating adrenal cortical and medullary tumors: an immunohistochemical study. Mod Pathol 16: 591-597, 2003.

26. Pan CC, Chen PC, Tsay SH and Ho DM: Differential immunoprofiles of hepatocellular carcinoma, renal cell carcinoma, and adrenocortical carcinoma: a systemic immunohistochemical survey using tissue array technique. Appl Immunohistochem Mol Morphol 13: 347-352, 2005. 